\title{
TERAPIA COMUNITÁRIA INTEGRATIVA: SITUAÇÕES DE SOFRIMENTO EMOCIONAL E ESTRATÉGIAS DE ENFRENTAMENTO APRESENTADAS POR USUÁRIOS
}

\author{
Ianine Alves da ROCHA , Aralinda Nogueira Pinto de SÁ , Lucineide Alves Vieira BRAGA , \\ Maria de Oliveira FERREIRA FILHA ${ }^{\mathrm{d}}$, Maria Djair DIAS
}

\section{RESUMO}

A Terapia Comunitária Integrativa (TCI) surge como uma estratégia de apoio à saúde mental dos usuários do Sistema Único de Saúde. O estudo tem como objetivo identificar os principais problemas apresentados pelos usuários da terapia comunitária e identificar as estratégias que utilizam no enfrentamento das situações que provocam sofrimento emocional. Pesquisa do tipo documental realizada na Secretaria Municipal de Saúde, tendo como fontes de informações fichas de organização de dados da TCI. Os resultados demonstraram que o problema mais frequente é o estresse, que a estratégia de enfrentamento mais utilizada é a espiritualidade, e que através dos discursos dos participantes é possível perceber a opinião positiva que eles têm dos encontros. A TCI é um espaço onde as relações construídas transmitem apoio emocional, fortalecem vínculos e diminuem os casos de exclusão social.

Descritores: Saúde mental. Atenção primária à saúde. Processo saúde-doença.

\section{RESUMEN}

La Terapia Comunitaria Integradora (TCI) se ha convertido en una estrategia de apoyo de salud mental a los usuarios del Sistema Nacional de Salud. El estudio tiene como objetivo identificar los principales problemas presentados por los usuarios de la atención comunitaria e identificar las estrategias que utilizan para afrontar las situaciones que causan estrés emocional. La investigación fue documental celebrada en el Municipal de Salud en el que las fuentes de información fichas para organizar los datos de la TCI. Los resultados mostraron que el problema más frecuente es el estrés, la estrategia de enfrentamiento más utilizada es la espiritualidad, y a través de los discursos de los participantes es posible percibir la connotación positiva que tienen las reuniones. La TCI es un espacio donde las relaciones construidas transmiten apoyo emocional, fortalecen los vínculos y reducen los casos de exclusión social.

Descriptores: Salud mental. Atención Primaria de Salud. Proceso de salud-enfermedad.

Título: Terapia comunitaria integradora: situaciones de angustia emocional y estrategias de afrontamiento presentadas por los usuarios.

\section{ABSTRACT}

Community Integrative Therapy (CIT) has emerged as a strategy to support the mental health of users of the public Unified Health System (SUS). This study had the objective to identify the principal problems presented by patients of group therapy, and to identify strategies that they use to cope with situations that cause emotional suffering. Secondary research carried out at the Municipal Secretary of Health, with information sources being organization forms with data from CIT. The results demonstrate that the most frequent problem [cause of suffering] is stress, the coping strategy most used is spirituality, and through the discourses of the participants it is possible to perceive the positive perception that they have of the meetings. CIT is a space where the relationships that are forged transmit emotional support, strengthen ties and diminish cases of social exclusion.

Descriptors: Mental health. Primary health care. Health-illness process.

Title: Community integrative therapy: situations of emotional suffering and patients' coping strategies.

\footnotetext{
a Enfermeira da Secretaria Estadual de Saúde. Mestre em Enfermagem pela Universidade Federal da Paraíba -UFPB. João Pessoa/PB. Brasil. E-mail: ian_ine@yahoo.com.br.

b Enfermeira da Secretaria Estadual de Saúde. Mestre em Enfermagem pela UFPB. João Pessoa/PB. Brasil. E-mail: arallinda@yahoo.com.br. c Enfermeira da Secretaria Municipal de Saúde Mestre em Enfermagem pela UFPB. João Pessoa/PB. Brasil. E-mail:lucineide.avb@gmail.com.

d Enfermeira. Doutora em Enfermagem. Docente do Programa de Pós-Graduação em Enfermagem pela Universidade Federal Paraíba. Líder do Grupo de Estudos e Pesquisa em Saúde Mental Comunitária. João Pessoa, PB. Brasil. E-mail: marfilha@yahoo.com.br.

e Enfermeira,Doutora. Docente do Programa de Pós-Graduação em Enfermagem na UFPB. Vice-Lider do Grupo de Estudos e Pesquisa em Saúde Mental Comunitária. Brasil. E-mail: mariadjair@yahoo.com.br.
} 


\section{INTRODUÇÃO}

O modelo de assistência de base comunitária, que enfoca o cuidado na família, vem se delineando desde 1994. Este modelo considera o meio social, as atitudes da vida moderna e a promoção da saúde como seus fundamentos básicos. Deste modo, foi surgindo no processo de cuidado a valorização de conceitos como acolhimento e humanização, que apontam a solução dos problemas por meios não convencionais até então, mas pelo diálogo, fé, grupos de apoio e outras formas de promover o bem-estar, ressalvando a posição da família como importante instrumento de cuidado de seus membros. Tudo isso faz com que o processo saúde-doença receba atenção especial, criando vínculos de responsabilidade entre os sujeitos comunitários o que contribui para o fortalecimento da saúde mental da população.

Nesse sentido, a Terapia Comunitária Integrativa (TCI) emerge como uma estratégia de inclusão social e apoio à saúde mental da população, que é definida como um espaço de acolhimento, para a partilha de sofrimentos e sabedoria de vida, que ocorre de maneira circular e horizontal. Constitui um espaço de escuta, reflexão e troca de experiências, criando uma teia de relação social entre os participantes, na busca de soluções para os conflitos pessoais e familiares apresentados no encontro ${ }^{(1)}$.

A TCI é uma tecnologia de cuidado, que tem dado respostas satisfatórias aos que dela se beneficiam, sendo mais um instrumento de trabalho, que pode ser utilizado pelos profissionais de saúde no enfrentamento de situações de sofrimento advindas do cotidiano. Através da terapia, estes profissionais e as pessoas da comunidade envolvidas podem compreender melhor a origem de seus problemas e desenvolver estratégias que permitem um direcionamento de ações para a promoção da saúde, conforme entendida na carta de Ottawa (1986); e melhora da qualidade de vida da população( ${ }^{(2)}$.

Considerando a relevância da Terapia Comunitária Integrativa para a saúde mental e em detrimento da carência de investigação acerca desta, surgiram os seguintes questionamentos: Quais as situações de sofrimento emocional mais frequentes nas rodas de TCI? Quais as estratégias de enfrentamento mais utilizadas por usuários da TCI?

Tendo em vista que o conhecimento desses problemas empodera a conduta dos profissionais de saúde envolvidos nas rodas, o estudo possui os seguintes objetivos: identificar situações de sofrimento emocional mais frequentes nos relatos de usuários da TCI; e identificar as estratégias de enfrentamento mais utilizadas diante de situações que provocam sofrimento emocional, relatadas por usuários da TCI.

\section{MÉTODOS}

Trata-se de uma pesquisa documental de caráter descritivo realizada no Município de João Pessoa na Paraíba, que teve como fonte de investigação fichas de organização das informações da Terapia Comunitária Integrativa, as quais contêm relatos descritivos das terapias realizadas no ano de 2008.

A coleta de dados ocorreu em etapas durante o período de setembro de 2008 a maio de 2009. A primeira foi marcada pelo acesso aos arquivos da TCI na Secretaria Municipal de Saúde, no setor de Atenção Básica de Saúde. Após a consulta, as fichas foram agrupadas de acordo com a classificação distrital (Distrito Sanitários: I, II, III, IV e V) e sequenciadas de acordo com o mês de realização da terapia. No final da organização foram catalogadas 776 fichas contendo informações sobre o número de participantes, a faixa etária, os principais temas relatados, as estratégias de enfrentamento das situações de sofrimento e depoimentos espontâneos dos participantes da terapia acerca do momento vivenciado.

Depois da leitura de cada ficha, as situações problemas encontradas foram inicialmente compiladas sob aspectos de categorias, como propõe o instrumento que avalia o impacto da $\mathrm{TCI}^{(1)}$; em seguida, as estratégias para o enfrentamento das situações que provocam sofrimento emocional foram organizadas em um banco de dados com informações descritas na forma de variáveis. Os achados foram analisados de maneira discursiva à luz da literatura pertinente. Convém compreender que se adotou como principal situação problema de cada ficha aquela que foi selecionado na roda de terapia como tema mote, ou seja, o tema que foi apresentado por um dos usuários e escolhido para a problematização; e que todas as estratégias de enfrentamento citadas foram consideradas neste estudo. Aos resultados encontrados, optou-se por discutir apenas aqueles que tiveram maiores destaque.

Esta pesquisa obedeceu às normas e às diretrizes previstas na Resolução N. 196/1996, que 
regulamenta as pesquisas com seres humanos e foi submetida à aprovação pelo Comitê de Ética do Centro de Ciências da Saúde da Universidade Federal da Paraíba, do qual obteve parecer favorável, com protocolo número 021/0607.

\section{RESULTADOS E DISCUSSÃO}

Deste estudo emergiram três categorias: o estresse que representa situações diversas do dia a dia geradoras de sensações de cansaço físico, esgotamento mental e desanimo; os conflitos familiares relacionados aos diversos entes, causadores de sofrimento, instabilidade e insegurança familiar; e a categoria trabalho relativo à ausência deste ou à situações potenciais de conflitos no trabalho. A tabela a seguir mostra o quantitativo das diversas categorias encontradas:

\section{A dor que revela o sofrimento no cotidiano}

Os principais temas apresentados foram distribuídos em um dos instrumentos de avaliação dos impacto da $\mathrm{TCI}^{(1)}$. Destaca-se que os temas apresentados na tabela 1 são oriundos dos relatos de participantes da TCI em sua grande maioria têm residências em bairros pobres ou comunidades carentes, os quais refletem a realidade vivenciada pela população local. Os dados nos mostram que o sofrimento mais presente na população é o estresse que, segundo a proposta do idealizador da TCI, se agregam a essa categoria registros que revelem situações de cansaço, medo, ansiedade, desamparo, desmotivação, os quais podem levar cada vez mais ao nervosismo e a insônia comprometendo a qualidade de vida dos indivíduos.

$\mathrm{O}$ adoecimento mental se tornou a responsável pela maioria dos males que aflige a sociedade, principalmente os relacionados ao estilo de vida urbano atual. A preocupação científica com essa questão reside na sua provável relação com o adoecimento ou sofrimento psíquico, como confusão, perda do senso de humor, ansiedade, nervosismo, depressão, raiva, frustração, preocupação, medo, irritabilidade e impaciência ${ }^{(3)}$. E essa cascata de problemas pode ser facilmente identificada nas falas registradas nas fichas:

Tabela 1 - Distribuição dos principais temas relatados pelos usuários durante os encontros de Terapia Comunitária Integrativa. João Pessoa, PB, 2009.

\begin{tabular}{lcc}
\hline Temas dos encontros de TCI & N & \% \\
\hline Estresse & 229 & 29,51 \\
Conflitos Familiares & 110 & 14,17 \\
Trabalho & 84 & 10,82 \\
Problemas de saúde & 80 & 10,3 \\
Depressão/perda & 66 & 8,5 \\
Violência & 43 & 5,54 \\
Alcoolismo & 40 & 5,15 \\
Abandono & 25 & 3,22 \\
Drogadição & 24 & 3,09 \\
Solidão & 20 & 2,57 \\
Partilha de alegria & 19 & 2,44 \\
Conflitos & 12 & 1,54 \\
Rejeição & 10 & 1,28 \\
Discriminação & 7 & 0,9 \\
Deficiências Mentais & 5 & 0,64 \\
Prostituição & 2 & 0,25 \\
\hline TOTAL & 776 & $100 \%$ \\
\hline
\end{tabular}

Fonte: Material empírico do estudo, 2009. 
Sinto angústia por ter contas a pagar [...]. (F1)

Sinto pânico e vontade de chorar por não ter criado minha filha $[\ldots] \cdot(F 2)$

Estou triste pela ausência do meu irmão [...]. (F3)

$\mathrm{O}$ conceito de estresse tem sido amplamente utilizado nos dias atuais, chegando mesmo a tornar-se parte do senso comum passando a ser responsável por quase todos os males que afligem as adversidades do dia a dia, principalmente em decorrência da vida moderna. Em vista disso, não é de se espantar que tenha havido um crescimento de terapêuticas e de programas voltados para o controle do estresse, com ênfase no crescimento do interesse econômico em torno do estresse, que pode ser observado tanto na indústria farmacêutica. O que sugere, neste estudo, atribuir o termo estresse ao senso comum e sua visão biopsicossocial, que considera os estímulos estressores provenientes tanto do meio externo (estímulos de ordem física ou social, como o trabalho), quanto do interno (pensamentos, emoções, fantasias e sentimentos, como angústia, medo, alegria e tristeza) ${ }^{(4)}$.

Estudos mostram que no Brasil o estresse é, em $70 \%$ dos casos, a causa de procura de serviços médicos sendo considerado uma grave questão socioeconômica e de saúde pública. Tais estudos destacaram as influências biopsicossociais que interferem no bem-estar psicológico e as relações que a permeiam com o propósito de melhor compreender o processo de adoecimento mental ${ }^{(5)}$.

Essa correria do cotidiano causadora de estresse reflete diretamente no relacionamento familiar gerando conflitos, onde os membros da família pressionados pelos sistemas social e econômico vigente, já não se sentam juntos às refeições, não perguntam sobre como passaram seus dias, não dialogam sobre seus problemas, fazendo com que a familiar venha perdendo suas funções para escolas, meios de comunicação, o seu lugar de detentora de transmissão cultural de valores e normas, e interação social de seus membros.

Na sociedade atual, atribui-se ao processo de industrialização e urbanização a formação de famílias extensas e com vínculos frágeis, uma vez que pela ocupação de homens e mulheres no mercado de trabalho e sua crescente carga horária tornou-se necessária à presença de outros parentes no domicílio, o que implicou na diminuição do entrosamento entre eles $^{(6)}$. $́$ É assim que os valores tradicionais que regiam as famílias, como o respeito, o amor e o incentivo vêm se dissolvendo cada vez mais.

Este fato está representado pela percentagem do segundo tema mais indicado: conflitos familiares, que foram apresentados nos encontros de TCI com frases assim:

\section{Minha filha não me respeita $[\ldots ..] \cdot(F 4)$}

Descobri que meu marido tem outra […]. (F5)

Estes conflitos podem estar diretamente relacionados ao índice elevado de sofrimento mental, pois o ser humano precisa do apoio familiar para se sentir socialmente completo. Assim, nos dias atuais, ainda se considera importante o papel da família, pois é a única que pode desempenhar a socialização dos membros e a estabilização do equilíbrio da personalidade adulta ${ }^{(6)}$. Dessa forma, as pessoas têm a família como uma referência fundamental na vida, no sentido de experiência de felicidade, suporte e promoção de equilíbrio pessoal. Entretanto, ela também está associada a experiências de conflitos entre os membros, preocupações, solidão e sofrimento ${ }^{(7)}$.

A família deverá ser encarada como um todo que integra contextos mais vastos como a comunidade em que se insere, sendo, portanto, um sistema de membros interdependentes que possuem dois atributos: comunidade dentro da família e interação com outros membros ${ }^{(8)}$. Cumpre assinalar, que em toda família coexistem tendências para saúde e para doença, o diferencial se fará a depender de como a família enfrenta situações de crise, de como está a afetividade e a comunicação entre seus componentes. Estes serão indicadores de relações saudáveis ou adoecidas ${ }^{(9)}$.

A TCI enquanto instrumento que permite ser desenvolvido com a história de vida de participantes, tem revelado situações problemas que caracterizam a realidade da comunidade envolvida, problemas estes que seguem como uma cascata sistêmica que pode ser resultado do estreitamento de relações, principalmente, aquelas entre os membros que compõe uma família.

\section{Com força e fé na vida: estratégias de enfrentamento da dor}

Nos encontros de TCI as situações problemas escolhidas despertavam várias estratégias de 
enfrentamento resgatadas pelas as experiências de vida de cada sujeito com sua essência resiliente. Do total de 776 encontros, foram registradas 1.379 estratégias de enfrentamento, e todas foram consideradas e agrupadas por semelhança de tema à medida que iam sendo mencionadas. As principais estratégias foram: espiritualidade (fé/oração), diálogo, apoio familiar, apoio de amigos, perdão, apresentar força de vontade/determinação (ter perseverança e coragem diante das dificuldades), outros (contempla-se aqui oito grupos de estratégias de menor representação numérica).

Como mostram os dados, a estratégia mais utilizada foi o fortalecimento da espiritualidade. Tal temática tem merecido destaque na área da saúde por existir, hoje, um reconhecimento da medicina de que a saúde de indivíduos é determinada pela interação de fatores físicos, mentais, sociais e espirituais. A espiritualidade pode ser definida como um sistema de crenças que transmite vitalidade e significado a eventos da vida; ela pode mobilizar energias e iniciativas extremamente positivas, com potencial ilimitado para melhorar a qualidade de vida da pessoa. Há relação entre envolvimento espiritualista e vários aspectos da saúde mental, sendo que pessoas vivenciam melhor a saúde mental e se adaptam com mais sucesso ao estresse quando são religiosas ${ }^{(10)}$.

No cenário da TCI, frequentemente, a espiritualidade é associada à religiosidade e à fé. É notório observar que as pessoas religiosas parecem lidar mais facilmente com os estresses da vida, recuperam-se mais rapidamente de depressão e apresentam menos ansiedade e outras emoções negativas do que as pessoas menos religiosas. Dessa forma, o conhecimento do impacto positivo que a espiritualidade promove pode ser valorizado pelos profissionais para lidarem melhor com o sofrimento mental dos pacientes, visto que a maioria das pesquisas feitas com populações saudáveis sugere que as crenças e as práticas religiosas estão associadas a maior bem-estar, melhor saúde mental e a um enfrentamento mais exitoso de situações estressantes $^{(11)}$.

Sob esse prisma, a espiritualidade pode proporcionar aumento do significado da vida, o que está associado à maior resiliência e resistência ao estresse relacionado ${ }^{(12)}$. Nessa perspectiva, as pessoas que seguem uma religião e/ou têm uma espiritualidade considerável podem desenvolver com mais facilidade, sentimentos de perdão, que corresponde a uma das estratégias de enfrentamento mais utilizada por parte dos usuários da TCI.

Cumpre assinalar que o perdão corresponde ao processo mental ou espiritual de absorver da pena por uma culpa, respondendo com graça a um ressentimento ou raiva contra outra pessoa. Além disso, todo ato de perdão tem lugar na humildade e exige confiança. Ele é um processo e, como tal, acontece em etapas de maior ou menor profundidade, que começa com a decisão de não se vingar, rompendo as engrenagens da violência e negando-se a combater com as armas do ódio ${ }^{(13)}$. O saber perdoar pode diminuir o processo de sofrimento emocional e físico, evitando a permanência de uma situação de estresse; com ele se pratica, também, a

Tabela 2 - Estratégias de enfrentamento sugeridas durante os encontros de Terapia Comunitária Integrativa entre idosos. João Pessoa, PB, 2009.

\begin{tabular}{lcc}
\hline Estratégias de enfrentamento & $\mathbf{N}$ & $\mathbf{\%}$ \\
\hline Espiritualidade & 390 & 28,28 \\
Perdão & 220 & 15,95 \\
Diálogo & 121 & 8,77 \\
Apoio familiar & 115 & 8,33 \\
Determinação & 112 & 8,12 \\
Apoio de amigos & 94 & 6,81 \\
Outros & 327 & 23,66 \\
\hline TOTAL & 1.379 & $100 \%$ \\
\hline
\end{tabular}

Fonte: Material empírico do estudo, 2009 
capacidade de compreender as atitudes do próximo e as situações vividas, além de desenvolver sentimentos de aceitação, conformação e paciência na espera de soluções. Isso gera nas relações um clima de paz e de felicidade interior.

O diálogo foi outra estratégia de enfrentamento destacada, por meio do qual se revela e soma a experiência e a intuição das pessoas num processo de respiração de idéias. No modo de vida atual, precisa-se do acúmulo de experiência de todos os povos para se ultrapassar os problemas que se criam e ameaçam a vida.

Com base nesse entendimento, o diálogo é uma relação de comunicação, que gera a crítica e a problematização, já que ambos os parceiros podem perguntar: por quê? Quem dialoga, dialoga com alguém e sobre algo, fazendo com que os sujeitos sejam atuantes no processo rumo à conscientização ${ }^{(14)}$. Isso acontece devido o diálogo ocorrer na igualdade em que todos procuram pensar e agir criticamente; para isso, exige-se humildade; supõe-se paciência; traduz-se a fé na historicidade de todos os homens, como construtores do mundo e implica na esperança de que sejam vislumbrados meios de tornar o amanhã melhor para todos.

A teoria da comunicação enquanto pilar teórico da TCI chama a atenção para o fato de que a comunicação entre pessoas é elemento que une os indivíduos, a família e a sociedade; e que para compreender os significados de sua transmissão é necessário considerar o comportamento dos indivíduos, a relação entre os que estão se comunicando e a linguagem utilizada, em que esta passa a ser a cultura, e a palavra é o instrumento para que o homem se torne homem ${ }^{(1-14)}$.

A Terapia Comunitária se tornou relevante instrumento de desabafo e alívio dos sofrimentos, numa perspectiva resiliente para todos que venham a passar por situações diversas que fragilizam a família, em decorrência dos fatores responsáveis pela perda dos papeis familiares, pelo rompimento dos vínculos afetivos, pelo desrespeito entre os membros que culminam na ausência do diálogo, na dissolução de sentimento, que levam a traição dos cônjuges e até mesmo a separação matrimonial ${ }^{(15)}$.

As rodas de TCI possibilitam que as pessoas percebam criticamente a sua realidade e necessidades, desenvolvendo potencialidades e buscando em conjunto os meios para resolução de seus problemas de forma consciente e efetiva.

\section{Aprendendo com a minha história e com a história do outro}

As ações de compartilhar as experiências instigam a autonomia dos sujeitos e permite que o indivíduo se sinta protagonista na busca da superação de suas dificuldades, o que caracteriza a capacidade resiliente de cada participante. Resiliência é um processo dinâmico que resulta na adaptação positiva em contextos de grande adversidade, independente do fator socioeconômico e inteligência, apresentando o papel de desenvolver, ao longo de todo ciclo da vida, a habilidade no indivíduo de sair transformado e fortalecido frente às situações diversas, contribuindo para a qualidade de vida(16).

A resiliência comunitária depende do aspecto cultural e valorativo que afetam o processo de adaptação positiva de cada sociedade. Ela funciona como ferramenta que ajuda os indivíduos e grupos a se beneficiarem das experiências vividas, de modo que a dificuldade possa significar o desafio para mobilizar as capacidades solidárias da população, fazendo assim um chamado à responsabilidade coletiva.

Diante destas ponderações, os registros nas Fichas de Organização das Informações da TCI apontam a contribuição deste tipo de terapia como ferramenta que valoriza o poder resiliente existente em cada participante, visto que o trabalho em grupo fortalece as potencialidades individuais e grupais, de modo que amplia a visão de recursos disponíveis na busca de novas estratégias para enfrentamento de desafios. Nos depoimentos espontâneos dos participantes pode-se destacar algumas falas registradas que revelam os sentimentos dos mesmos em relação à experiência vivida:

Encontrei uma outra família; Saio muito aliviada $[\cdots] \cdot(D 1)$.

Sei que não estou sozinha. (D2)

Feliz por ter encontrado um grupo de amigas [...]. (D3)

Me sinto bem participando desse encontro. (D4)

Quando venho aqui me sinto de forças recarregadas $[\cdots] \cdot(\mathrm{D} 5)$

Saio daqui com a sensação de que tudo vai dar certo $[\cdots] \cdot(D 6)$ 
Percebe-se nos depoimentos a importância da TCI para a conquista da autoestima, do bem estar, que caracterizam o conforto, a gratidão e a diminuição de sofrimentos angustiantes. Cada encontro acontece de maneira única, os problemas se repetem, contudo as experiências são sempre inovadoras, fazendo com que haja a construção de um saber novo frente às histórias vividas.

Portanto, o espaço do grupo pode facilitar o encontro entre pares, na medida em que, propiciando este mostrar-se igual, supera-se o individualismo presente até então. Assim, o grupo torna-se a maneira mais adequada para aprofundar o conhecimento, que se funda nos homens como corpos conscientes e na consciência como consciência intencionada ao mundo. A transmissão do saber não pode ser como depósito de conteúdos, mas como problematização dos homens em suas relações com o mundo ${ }^{(17)}$.

A prática da TCI tem evidenciado que a socialização e o compartilhamento das situações de sofrimento tem sido um veículo poderoso na sedimentação da Resiliência das pessoas envolvidas nos grupos dessa terapia $^{(18)}$.

Compreendendo que a vivencia pessoal e o compartilhamento de sofrimentos são fontes de saber, as reflexões dos encontros de TCI são necessárias uma vez que funcionam como mola propulsora para o processo de empoderamento e resiliência ${ }^{(17)}$. Assim, torna-se essencial a adoção cada vez maior dessas práticas nas Unidades de Saúde da Família de modo a possibilitar a participação do sujeito de maneira ativa na busca de uma melhor qualidade de vida.

\section{CONSIDERAÇÕES FINAIS}

Com a oportunidade de ampliar a leitura durante esse estudo, foi compreendido que a saúde mental de base comunitária vem crescendo no Brasil, e a TCI vem contribuindo para isto enquanto instrumento de cuidado que auxilia na prevenção do adoecimento mental e na promoção da saúde mental e geral. Investimentos como esses são destinados a toda a população e vêm mostrando resultados satisfatórios, conforme mostra os registros de falas. Isso serve de estímulo para que a visão holística oportunizada pela TCI ocupe cada vez mais espaço na atenção à saúde e amplie-se a oferta de serviços mais humanizados.

A pesquisa revelou que os principais problemas relatados nas rodas de TCI no município de
João Pessoa na Paraíba estão relacionados ao estresse, a problemas de ordem familiares e de trabalho, que, juntos, perfizeram $54,4 \%$ dos temas relatados; e que as estratégias de enfrentamento usadas pelos participantes são o diálogo, o desenvolvimento de atitudes de perdão e o fortalecimento da espiritualidade, que totalizaram $44,2 \%$ das estratégias.

Os registros de falas mostram os efeitos dos encontros, e demonstram a importância da TCI como uma estratégia de promoção da saúde mental que auxilia o trabalho das Equipes de Saúde da Família, por permitir compreender os conflitos existentes nas famílias, traçando assim condutas com maior poder de resolutividade. Não se pode negligenciar que a TCI é um espaço onde as relações construídas transmitem apoio emocional, fortalecem vínculos, diminuem os casos de exclusão social, e instiga a capacidade resiliente individual e grupal. É uma ferramenta de cuidado de baixo custo, cujos resultados positivos que vêm sendo alcançados pela comunidade devem ser divulgados para o fortalecimento da mesma enquanto estratégia de cuidado. Por essa relevância, há possibilidades de pesquisas que apontem para os benefícios da TCI aos distintos grupos: idosos, mulheres, adultos e crianças.

\section{REFERÊNCIAS}

1 Barreto AP. Terapia comunitária: passo a passo. Fortaleza: LCR; 2008.

2 Souza GML, Silva PMC, Azevedo EB, Ferreira Filha MO, Sila VCL, Espinola LL. A contribuição da terapia comunitária no processo saúde - doença. Cogitare Enferm. [Internet]. 2011 [citado 2013 Mar 15]:16(4):682-8. Disponível em: http:// ojs.c3sl.ufpr.br/ojs2/index.php/cogitare/article/ view/23030/17059.

3 Pascoal FFS. Síndrome de Burnout entre os profissionais de saúde da Estratégia Saúde da Família: risco de adoecimento mental [dissertação]. João Pessoa (PB): Programa de Pós-Graduação em Enfermagem, Centro de Ciências da Saúde, UFPB; 2008.

4. Sadir MA, Bignotto MM, Lipp MEN. Stress e qualidade de vida: influência de algumas variáveis pessoais. Paideia [Internet]. 2010 [citado 2012 Nov 19];20(45):73-81. Disponível em: http://www.scielo. $\mathrm{br} / \mathrm{pdf} /$ paideia/v20n45/a10v20n45.pdf.

5 Miranda CA, Tarasconi, CV, Scortegagna SA. Estudo epidêmico dos transtornos mentais. Aval Psicol 
[Internet]. 2008 [citado 2013 Mar 01]:17(2):249-57. Disponível em: http://pepsic.bvsalud.org/pdf/avp/ v7n2/v7n2a15.pdf.

6 Serapioni MO. Papel da família e das redes primárias na reestruturação das políticas sociais. Fortaleza: Ciênc Saúde Coletiva [Internet]. 2005 [citado 2011 Jan 20]. Disponível em: http:/ / redalyc.uaemex.mx/ pdf/630/63009925.pdf.

7 Cardoso CL, Féres-Carneiro TF. Sobre a família: com a palavra, a comunidade. Estud Pesqui Psicol [Internet]. 2008. [citado 2013 Jan 15];8(2),511526. Disponível em: http://pepsic.bvsalud.org/pdf/ epp/v8n2/v8n2a25.pdf.

8 Dias MO. Um olhar sobre a família na perspectiva sistemica no processo de comunicação no sistema familiar. Gest Desenvolv [Internet] 2011 [citado 2013 Mar 16]. Disponível em: http://repositorio. ucp.pt/bitstream/10400.14/9176/1/gestaodesenvolvimento19_139.pdf.

9 Mendes EV. O cuidado das condições crônicas na atenção primária à saúde: o imperativo da consolidação da estratégia da saúde da família. Brasília: Organização Pan-Americana da Saúde; 2012.

10 Murakami R, Campos CJG. Religião e saúde mental: desafio de integrar a religiosidade ao cuidado com o paciente. Rev Bras Enferm [Internet]. 2012 [citado 2013 Mar 10]: 65(2): 361-67. Disponível em: http:// www.scielo.br/pdf/reben/v65n2/v65n2a24.pdf

11 Koenig HG. Religião, espiritualidade e psiquiatria: uma nova era na atenção à saúde mental. Rev Psiquiatr Clín [Internet] 2007 [citado 2010 Maio 20];34(1):5-7. Disponível em: http://www.hcnet.usp. $\mathrm{br} / \mathrm{ipq} /$ revista/vol34/s1/index.html.

12 Delaney C, Barrere C. The influence of a spirituality-based intervention on psycho-spiritual outcomes in a cardiac population. Holist Nurs Pract [Internet]. 2008 [citado 2013 Maio 20];22(4):210-19. Disponível em:

\section{Endereço do autor / Dirección del autor / Author's address}

Ianine Alves da Rocha

Campus Universitário I, Jardim Cidade Universitária, CCS/PPGEnf

58059-900, João Pessoa, PB

E-mail:ian_ine@yahoo.com.br http://www.nursing2007 criticalcare.com/pt/ re/nestle/abstract.00004650-20080700000007. htm;jsessionid=RFPVxj9QLdLd8Foqcw ZQzy 1SZqlVqLLkn977pTMs 79cp5Z7Gkm QF!801907522!181195629!8091!-1.

13 Rodrigues W. Prolegômenos para uma fenomenologia do perdoar. Filosofix [Internet] 2010 [atualizado 2010 Set 08, citado 2010 Set 08]. Disponível em: http://www.filosofix.com.br/blogramiro/?p= 1735 .

14 Freire P. Pedagogia do oprimido. Rio de Janeiro: Paz e Terra; 2005.

15 Sá ANP, Rocha IA, Moraes MN, Braga LAV, Ferreira Filha MO, Dias MD. Conflitos familiares abordados na terapia comunitária integrativa. Rev Eletr Enferm [Internet]. 2012 [citado 2012 Nov 10];14(4). Disponível em: http://www.fen.ufg.br/revista/v14/ n4/v14n4-preview.htm.

16 Braga LAV, Dias MD, Ferreira Filha MO, Moraes MN, Araruna MHM, Rocha IA. Terapia comunitária e resiliência: história de mulheres. Rev Pesqui: cuid. fundam [Internet]. 2011 [citado 2012 Mar 01];3(5):251-68. Disponível em: http://bases.bireme.br/cgi-bin/wxislind.exe/iah/ online/? IsisScript $=\mathrm{iah} / \mathrm{iah} . \mathrm{xis} \& \mathrm{src}=$ google\&base $=$ BDENF\&lang $=$ p\&nextAction $=\operatorname{lnk} \&$ exprSearch $=2$ 3309\&indexSearch=ID.

17 Rocha IA, Braga LAV, Tavares LM, Andrade FB, Ferreira Filha MO, Dias MD, et al . A terapia comunitária como um novo instrumento de cuidado para saúde mental do idoso. Rev Bras Enferm [Internet]. 2009 [citado 2012 Nov 06];62(5):687-694. Disponível em: http://www. scielo.br/scielo.php?script $=$ sci_arttext\&pid $=$ So034$-71672009000500006 \& \operatorname{lng}=$ en. http://dx.doi. org/10.1590/So034-71672009000500006.

18 Camarotti MH, Gomes DO. Terapia comunitária: circularidade nas relações sociais. In: Osório LC, Valle MEP. Manual de terapia familiar. São Paulo: Artes Médicas; 2009.

Recebido em: 09.09.2011

Aprovado em: 28.05.2012 